\title{
28 Research Square \\ Recent findings on the role of STAT3 in breast cancer malignancy
}

Jia-hui Ma

Li Qin

Xia Li

\section{Video Byte}

Keywords: breast cancer, Signal transducer and activator of transcription 3, STAT3, chemotherapy, drug resistance, mammalian target of rapamycin, mTOR, cancer therapeutic, cancer prevention, Cell Communication and Signaling

Posted Date: October 27th, 2020

DOI: https://doi.org/10.21203/rs.3.rs-99171/v1

License: (c) (i) This work is licensed under a Creative Commons Attribution 4.0 International License.

Read Full License 


\section{Abstract}

Breast cancer is now the second leading cause of cancer-related deaths in women. However, because of its resistance to many cancer-fighting drugs, only a few treatment options are available. Emphasizing the need to discover and develop new methods for battling this disease. Signal transducer and activator of transcription 3 (STAT3) is known to contribute to breast cancer progression, proliferation, metastasis, and drug resistance. Making STAT3 a promising target for breast cancer therapy. A recent review reports what is currently known about the role of STAT3 pathways in breast cancer. and gives an update on the drugs currently available for targeting STAT3 pathways. New upstream regulators and downstream targets of STAT3 have been discovered and are potential drug targets, and combination therapy targeting mammalian target of rapamycin (mTOR) and STAT3 is a promising treatment option. Small-molecule inhibitors targeting STAT3, either alone or with other drugs, have also been found to be efficient therapeutic treatments. In all, recent studies suggest that STAT3 remains a strong clinical target for breast cancer prevention. Warranting further studies that will help identify and develop drugs that target this pathway. 